\title{
Increased growth factor expression after hepatic and pancreatic resection
}

\author{
CHRISTOPH JUSTINGER, CHRISTIAN SCHLÜTER, VILMA OLIVIERA-FRICK, \\ BERIT KOPP, CLAUDIA RUBIE and MARTIN K. SCHILLING
}

\author{
Klinik für Allgemein-, Viszeral- und Gefässchirurgie, Universitätskliniken des \\ Saarlandes, Geb. 57, D-66421 Homburg/Saar, Germany
}

Received January 21, 2008; Accepted April 18, 2008

DOI: $10.3892 /$ or_00000175

\begin{abstract}
Removal of the primary tumour is suggested to associate with an enhanced tumour growth of residual micrometastases. Recent data focus on growth factors that may be released in response to surgery-stimulating receptors of residual tumour cells. Vascular endothelial (VEGF) and hepatocyte growth factor (HGF) are potent inducers of angiogenesis. The two factors are necessary for wound healing and the promotion of tumour growth. This study was designed to determine growth factor serum levels in patients before, during and after major abdominal surgery. It was recently shown that simultaneous hepatic and pancreatic resection led to poor liver regeneration. As growth factors may be involved in these findings we compared the growth factor levels after liver resection with the levels in patients after pancreatic resection. Forty patients were accrued before hepatopancreatic surgery (hepatic resection $n=20$ and pancreatic resection $n=20$ ). Blood samples were taken from each patient immediately prior to surgery, during the operation and on the postoperative days (POD) 1-3, 5 and 10. To examine the wound fluid, liquid from the wound drains was collected on POD 3. Using ELISA the concentration of the angiogenic cytokines HGF and $\mathrm{VEGF}_{165}$ was determined. After the liver and pancreatic resections, circulating $\mathrm{HGF}$ and $\mathrm{VEGF}_{165}$ were increased. We found significantly higher levels of HGF on POD 1-3 ( $<<0.01)$, compared to preoperative results with a peak on POD 2 . After measuring the postoperative $\mathrm{VEGF}_{165}$ levels we found significantly higher levels of circulating VEGF $_{165}$ on POD 1-5 $(\mathrm{p}<0.01)$ compared to the preoperative levels. On comparing liver with pancreatic resection we did not detect significantly different levels of the two growth factors in the two groups. VEGF $_{165}$ and HGF concentrations measured during the operation demonstrated no change. HGF and $\mathrm{VEGF}_{165}$ levels
\end{abstract}

Correspondence to: Dr Christoph Justinger, Department of General, Visceral and Vascular Surgery, University of Saarland, D-66421 Homburg/Saar, Germany

E-mail: chcjus@uniklinik-saarland.de

Key words: growth factor, vascular endothelial growth factor, hepatocyte growth factor, liver resection, pancreatic resection detected in the wound fluid on POD 3 were $\sim 10$ times higher than the preoperative serum levels, respectively. In summary, our data show increased $\mathrm{VEGF}_{165}$ and HGF levels after hepatopancreatic surgery. Notably, the lack of an impact of the type of organ resection on the concentration-time curve of the two growth factors suggest that high postoperative growth factor levels are part of normal wound healing and systemic inflammation. Thus, the proangiogenetic potential of growth factors may account for accelerated tumour growth when residual tumour cells are exposed to high levels of $\mathrm{VEGF}_{165}$ and HGF.

\section{Introduction}

Primary and metastatic hepatic and pancreatic tumours are a common cause of death worldwide. Surgical treatment is the only curative option available for patients. Previous studies suggest that removal of the primary tumour can be associated with an enhanced tumour growth of residual micro-metastases (1). Experimental animal data show that metastatic growth after liver resection is significantly accelerated in the course of hepatic regeneration (2-5). However, the exact mechanism of this enhanced tumour growth remains unclear. Allendorf et al showed that cell-mediated immune function is affected after open surgery leading to increased tumour growth (6). Some authors have suggested that surgical excision may remove a variety of growth factor inhibitors resulting in angiogenesis and the subsequent growth of previously dormant metastases $(7,8)$. Recent data focus on growth factors released in response to surgery-stimulating receptors of residual tumour cells $(9,10)$.

Hepatocyte growth factor (HGF) is a mesenchymal cytokine with a number of biological activities including mitogenic and morphogenic properties in a variety of epithelial tissues. HGF is also known as an angiogenic factor that promotes endothelial cell growth, survival and migration $(11,12)$. It was first described in the blood of partially hepatectomized rats as a potent stimulator of growth in hepatocytes and neoplasms (13). The treatment of tumour cells in vivo with HGF results in an increase in the metastatic potential of the treated cells $(14,15)$.

Vascular endothelial growth factor (VEGF) is a potent mediator of angiogenesis inducing new vessel formation, endothelial cell proliferation and migration (16). Moreover, 
VEGF was shown to promote tumour growth (17). The release of VEGF and HGF after surgery may result in the promoted growth of residual tumour cells. Although increased plasma levels of growth factors were measured after gastric and colorectal surgery very few data report on the changes in serum and peritoneal fluid growth factor concentrations after hepatopancreatic surgery $(9,10,18)$. The aim of this study was to the determine peri- and postoperative $\mathrm{VEGF}_{165}$ and HGF levels in patients undergoing hepatic or pancreatic resection. Simultaneous hepatic and pancreatic resection leads to poor liver regeneration in animal models $(19,20)$. As growth factors may be involved in these findings we compared the growth factor levels after liver resections with the levels in patients after pancreatic resection.

\section{Materials and methods}

Patients. The study was approved by the local ethics committee of Saarland, Germany. Informed consent was obtained from all patients before study inclusion. Forty patients were accrued before hepatopancreatic surgery (20 patients before hepatic and 20 patients before pancreatic resection) (Table I) between May 2006 and July 2007. Information regarding surgical indication, clinical and pathological staging (cancer patients), demographics, intra- and postoperative course, time in ICU and in hospital were prospectively collected for all study patients (Table II). One blood sample was taken from each patient immediately prior to surgery and two samples were taken during the operation (one immediately after laparotomy and one after liver or pancreatic resection). In addition, six blood samples were taken on the postoperative days (POD) (one sample $1 \mathrm{~h}$ after the operation and POD 1-3, 5 and 10). To examine the wound fluid, liquid from the wound drains was collected on POD 3.

Exclusion criteria. Patients with liver cirrhosis (>Child A), chronic inflammatory diseases and patients with chronic viral infections such as hepatitis and HIV were not included. We excluded one patient after surgical revision within $24 \mathrm{~h}$ after the first operation.

Blood sampling and processing. Peripheral blood samples and wound fluid were collected in heparin-coated tubes. After being drawn plasma was isolated from samples by centrifugation at $1500 \mathrm{~g}$ for $5 \mathrm{~min}$ and then stored at $-80^{\circ} \mathrm{C}$ until ELISA assays were performed.

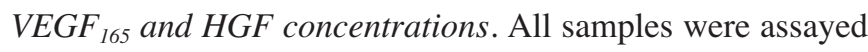
in duplicate using commercially available ELISA kits (Quantikine kit, R\&D Systems, Abingdon, Oxon, UK) following the manufacturer's guidelines.

Statistical analysis. All statistical analyses were performed using the SPSS software (sigma stat 3.0).

\section{Results}

Characteristics of patients and perioperative growth factor levels. Prior to hepatopancreatic surgery, 40 patients were enrolled in this study over a 14-month period. The median age
Table I. Type of operation.

\section{Liver resection}

Left hemihepatectomy

Right hemihepatectomy

Segmental resection ( $>1$ segment)

Pancreatic resection

Pylorus-preserving pancreaticoduodenectomy

'Classic' pancreaticoduodenectomy

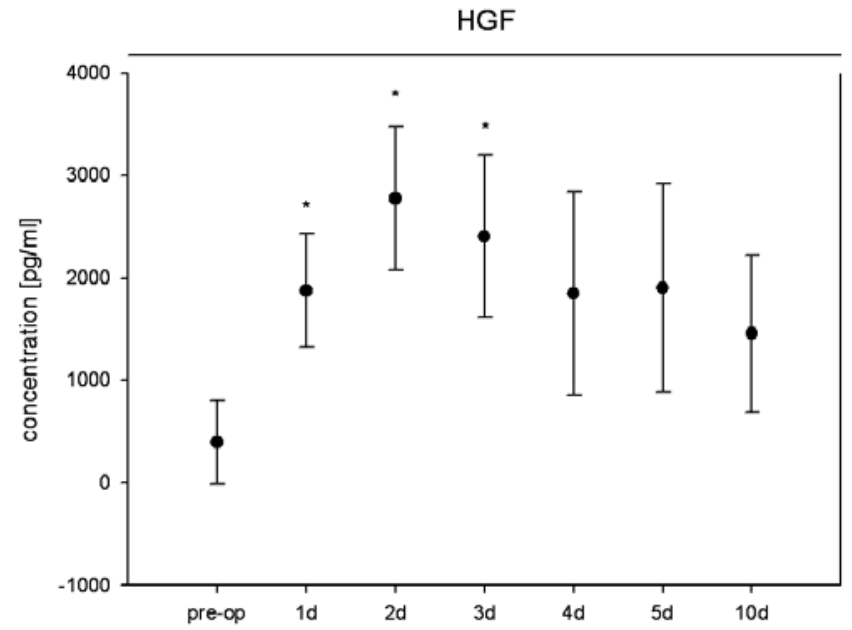

Figure 1. Concentration of serum HGF in patients undergoing hepatopancreatic surgery. Sampling points were preoperative (pre-op), and on postoperative days $1-5$ and 10. $\mathrm{p}<0.01$ (compared with preoperative levels).

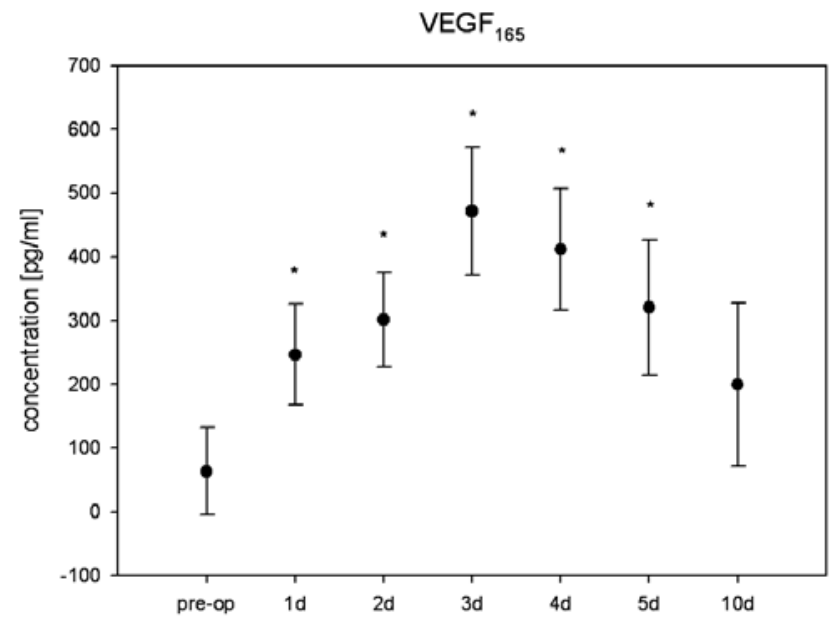

Figure 2. Concentration of serum VEGF in patients undergoing hepatopancreatic surgery. Sampling points were preoperative (pre-op), and on postoperative days $1-5$ and $10 .{ }^{*} \mathrm{p}<0.01$ (compared with preoperative levels).

of patients was 64 (35-82 years). There were 25 male (62.5\%) and 15 female patients. The demographic and operative data are shown in Tables I and II. 
Table II. Demographics and indication.

Hepatic resection

$\mathrm{N}$

Age (years)

BMI

ASA

Operation time (min)

ICU (days)

IHOS (days)

Indication

$\begin{array}{ll}\text { Metastatic colon carcinoma } & \mathrm{n}=15 \\ \text { Metastatic mamma carcinoma } & \mathrm{n}=1 \\ \text { HCC } & \mathrm{n}=3 \\ \text { Seminoma } & \mathrm{n}=1\end{array}$

Pancreatic resection

$216(138-330)$

$3.8(2-10)$

$13.4(7-28)$

Carcinoma pancreas

$\mathrm{n}=11$

Carcinoma duodenal papilla

$\mathrm{n}=4$

Cystadenoma

$\mathrm{n}=2$

Endocrine tumour

$\mathrm{n}=2$

Adenoma duodenal papilla

BMI, Body mass index; ASA, Physical status classification system by the American Society of Anesthesiologists (1963); ICU, Patients stay in the intensive care unit; IHOS, In hospital stay; HCC, Hepatocellular carcinoma.

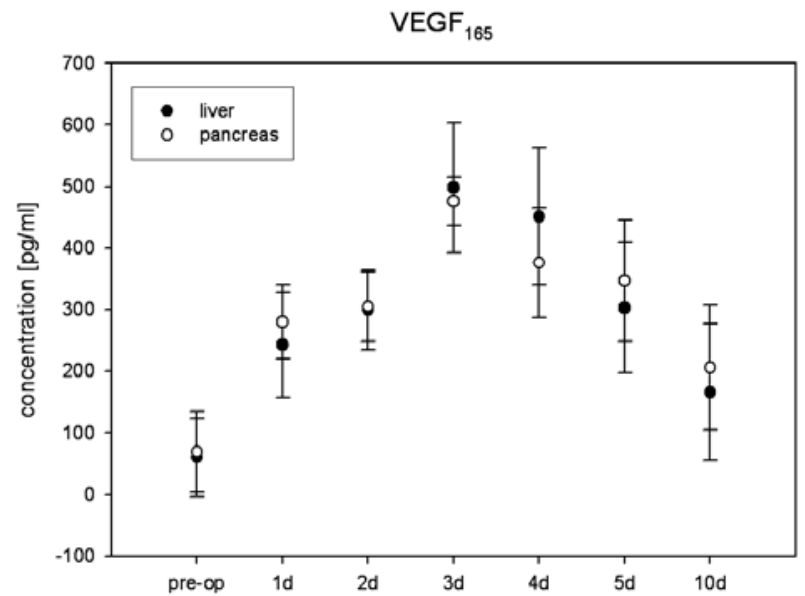

Figure 3. Concentration of serum VEGF in patients undergoing hepatic vs. pancreatic surgery. Sampling points were preoperative (pre-op), and on postoperative days $1-5$ and 10 . $\mathrm{p}>0.1$ (comparing the liver with the pancreas group).

The growth factor expression was evaluated in all patients before surgery. There was a great variation in the levels of the two growth factors before the operation (HGF: $394 \pm 424 \mathrm{pg} / \mathrm{ml}$ and $\mathrm{VEGF}_{165}: 63.4 \pm 68.3 \mathrm{pg} / \mathrm{ml}$ ). Measuring $\mathrm{VEGF}_{165}$ and HGF during the operation demonstrated no change in the growth factor concentrations (data not shown).

Postoperative levels of circulating factors. HGF and $\mathrm{VEGF}_{165}$ serum levels were examined postoperatively (Figs. 1 and 2). After the liver- and pancreatic resection, circulating HGF was increased with a peak at $48 \mathrm{~h}$. We found significantly higher levels of HGF on POD 1-3 compared to preoperative results ( $<<0.01$; POD 1: $1878 \pm 951$, POD 2: $2777 \pm 1237$ and POD 3: $2406 \pm 1127 \mathrm{pg} / \mathrm{ml})$. On POD 4, 5 and 10 we did not detect significantly higher plasma levels of HGF compared to the preoperative levels.

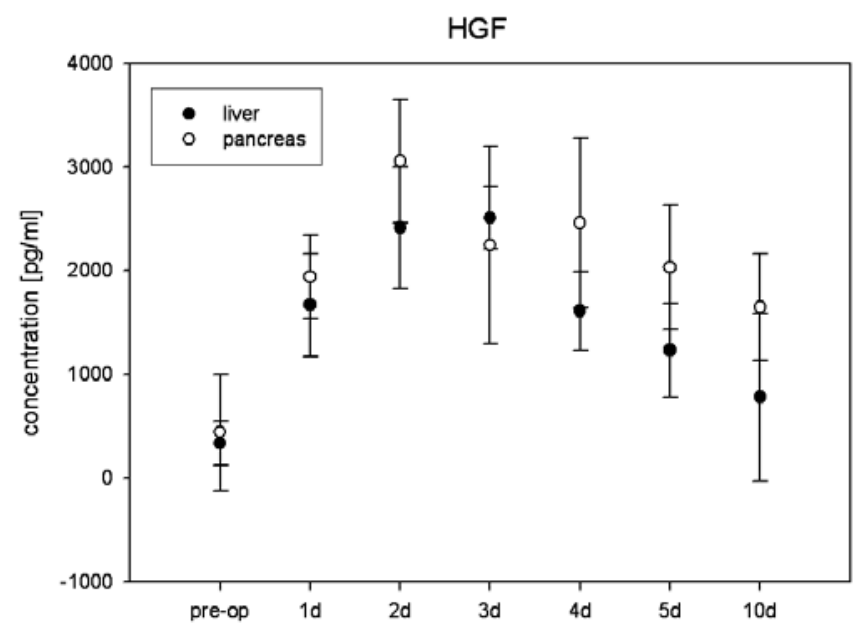

Figure 4. Concentration of serum HGF in patients undergoing hepatic vs. pancreatic surgery. Sampling points were preoperative (pre-op), and on postoperative days $1-5$ and $10 . p>0.1$ (comparing the liver with the pancreas group).

On measuring the postoperative $\mathrm{VEGF}_{165}$ levels we found significantly higher levels of circulating $\mathrm{VEGF}_{165}$ on POD 1-5 when compared with preoperative levels ( $<<0.01$; POD 1: 246 \pm 79 , POD 2: 301 \pm 74 , POD 3: 471 \pm 107 , POD 4: $411 \pm 138$ and POD 5: $320 \pm 106 \mathrm{pg} / \mathrm{ml})$. However, $\mathrm{VEGF}_{165}$ peaked on POD 3 and nearly returned to preoperative levels on POD 10.

Growth factors after liver and pancreatic surgery. To determine whether pancreatic surgery had an impact on HGF and VEGF levels we compared the results of the 20 patients after liver surgery to the 20 pancreatic resections. The two groups were nearly similar with regard to age, gender, body mass index and operation time (Table II). We did not detect significantly different levels of the two growth factors in the two groups (Figs. 3 and 4). 
$H G F$ and $V E G F_{165}$ levels in peritoneal fluid. We measured the concentration of HGF and $\mathrm{VEGF}_{165}$ in the wound fluid taken from the abdominal drains on POD 3. We found high levels of the two examined growth factors in the drain fluid (HGF: $4649 \pm 2140 \mathrm{pg} / \mathrm{ml}$ and $\mathrm{VEGF}_{165:} 804 \pm 215 \mathrm{pg} / \mathrm{ml}$ ). The detected HGF and $\mathrm{VEGF}_{165}$ levels in the wound fluid on POD 3 were $\sim 10$ times higher than the preoperative serum HGF and VEGF $_{165}$ levels.

\section{Discussion}

Experimental data suggest that surgical trauma may be associated with increased tumour growth (21-24,31). Various potential mechanisms have been identified demonstrating a correlation between surgical trauma and tumour growth $(6,25,26,31)$. However, these mechanisms were not linked to clinical outcome in cancer patients. Recent data focus on growth factors and angiogenesis and their influence on tumour growth. It is a well-known fact that normal wound healing is associated with angiogenesis and the release of proangiogenetic factors. This may be problematic for cancer patients as the proangiogenetic conditions may lead to accelerated tumour growth. This study aimed to elucidate whether surgical trauma may lead to elevated serum levels of growth factors.

Regardless of the surgical indication and organ resection, our studies demonstrated that major abdominal surgery was followed by a significant elevation of the two growth factors, VEGF and HGF, in serum and wound fluid. Few studies currently report on VEGF and HGF serum levels in the first 10 days after surgery.

VEGF plays a key role in wound healing (16). Therefore, serum VEGF levels may reflect the extent of wound healing after surgical traumas. The present study was the first to show there is no difference in $\mathrm{VEGF}_{165}$ serum levels when comparing liver and pancreatic surgery. Other studies reported that elevated $\mathrm{VEGF}_{165}$ levels after colorectal resections depended on the surgical method and incision length $(9,27)$. These observations suggest that it is the surgical trauma rather than the disease that is associated with increased $\mathrm{VEGF}_{165}$ levels.

HGF was the first growth factor described in the blood of partially hepatectomized rats and is a potent stimulator of growth in hepatocytes and neoplasms (13). There is increased synthesis of HGF by non-parenchymal cells after partial hepatectomy (28). In our investigation, we found increased HGF serum levels after liver and pancreatic resection. The serum HGF level changes in association with hepatocellular dysfunction, hepatic necrosis and systemic inflammation $(29,30)$. Systemic inflammation as a response to major abdominal surgery may explain the elevation of HGF after hepatic and pancreatic resection.

In summary, our data suggest that wound healing and systemic inflammation response after surgical trauma lead to increased VEGF and HGF levels. This may explain the elevation of the examined growth factors only for the first days after the operation. In addition, we detected high levels of HGF and $\mathrm{VEGF}_{165}$ in the drain fluid of our patients on POD 3. The observation that the growth-factor increase in drain fluid occurred regardless of the disease or type of surgical procedure suggests that it is a part of the normal early wound healing.
Since we did not find any impact of the type of operation and organ resection on the concentration-time curve of the two growth factors, our data do not explain the influence of pancreatic resection on liver regeneration.

Of note is what the elevated systemic levels of VEGF and HGF mean to cancer patients. It has been described that major surgery is associated with immunosuppression early after the surgical trauma (6). In this period no antiproliferative therapies such as chemotherapy are applied. However, the proangiogenetic potential of growth factors may account for accelerated tumour growth when residual tumour cells are exposed to high levels of VEGF and HGF. With a similar line of arguments Belizon et al see an alternative in antiangiogenetic therapy in the early postoperative period (9). It seems reasonable that elevated levels of proangiogenetic factors lead to accelerated tumour growth. In contrast, in the present study, major abdominal surgery was followed by significantly increased HGF and $\mathrm{VEGF}_{165}$ levels only for the first days after the operation. Except for these findings scarce data exist on serum growth-factor levels in the first two weeks after the trauma. Whether accelerated tumour growth for a few days has an impact on patient prognosis has to be discussed critically. As postoperative wound healing and regeneration is a complex process it is inevitable that additional factors will be involved. Therefore, continuous research will lead to a better understanding of the early postoperative period and the effect of the surgical trauma on tumour growth.

\section{Acknowledgements}

The authors would like to thank Mrs. Tiaré Serrano C. and Ms. Rabea Junker for their assistance.

\section{References}

1. Slooter GD, Marquet RL, Jeekel J and Ijzermans JN: Tumour growth stimulation after partial hepatectomy can be reduced by treatment with tumour necrosis factor alpha. Br J Surg 82: 129-132, 1995.

2. Mizutani J, Hiraoka T, Yamashita R and Miyauchi Y: Promotion of hepatic metastases by liver resection in the rat. Br J Cancer 65: 794-797, 1992.

3. van Dale P and Galand P: Effect of partial hepatectomy on experimental liver invasion by intraportally injected colon carcinoma cells in rats. Invasion Metastasis 8: 217-227, 1988.

4. de Jong KP, Lont HE, Bijma AM, et al: The effect of partial hepatectomy on tumor growth in rats : in vivo and in vitro studies. Hepatology 22: 1263-1272, 1995.

5. Rashidi B, An Z, Sun FX, et al: Minimal liver resection strongly stimulates the growth of human colon cancer in the liver of nude mice. Clin Exp Metastasis 17: 497-500, 1999.

6. Allendorf JD, Bessler M, Horvath KD, et al: Increased tumor establishment and growth after open versus laparoscopic surgery in mice may be related to differences in postoperative T-cell function. Surg Endosc 13: 233-235, 1999.

7. O'Reilly MS, Holmgren L, Shing Y, et al: Angiostatin: a novel angiogenesis inhibitor that mediates the suppression of metastases by a Lewis lung carcinoma. Cell 79: 315-328, 1994.

8. Folkman J: Angiogenesis inhibitors generated by tumors. Mol Med 1: 120-122, 1995.

9. Belizon A, Balik E, Feingold DL, et al: Major abdominal surgery increases plasma levels of vascular endothelial growth factor open more than minimally invasive methods. Ann Surg 244: 792-798, 2006.

10. Whitworth MK, Sheen A, Rosa DD, et al: Impact of laparotomy and liver resection on the peritoneal concentrations of fibroblast growth factor 2, vascular endothelial growth factor and hepatocyte growth factor. J Cancer Res Clin Oncol 132: 41-44, 2006. 
11. Bussolino F, Di Renzo MF, Ziche M, et al: Hepatocyte growth factor is a potent angiogenic factor which stimulates endothelial cell motility and growth. J Cell Biol 119: 629-641, 1992.

12. Grant DS, Kleinman HK, Goldberg ID, et al: Scatter factor induces blood vessel formation in vivo. Proc Natl Acad Sci USA 90: 1937-1941, 1993.

13. Nakamura T, Nawa K and Ichihara A: Partial purification and characterization of hepatocyte growth factor from serum of hepatectomized rats. Biochem Biophys Res Commun 122: 1450-1459, 1984.

14. Jiang WG, Lloyds D, Puntis MC, et al: Hepatocyte growth factor/ scatter factor, liver regeneration and cancer metastasis. $\mathrm{Br} \mathrm{J}$ Surg 80: 1368-1373, 1993.

15. Di Renzo MF, Narsimhan RP, Olivero M, et al: Expression of the Met/HGF receptor in normal and neoplastic human tissues. Oncogene 6: 1997-2003, 1991.

16. Neufeld G, Cohen T, Gengrinovitch S, et al: Vascular endothelial growth factor (VEGF) and its receptors. FASEB J 13: 9-22, 1999.

17. Jung YD, Nakano K, Liu W, et al: Extracellular signal-regulated kinase activation is required for up-regulation of vascular endothelial growth factor by serum starvation in human colon carcinoma cells. Cancer Res 59: 4804-4807, 1999.

18. Ikeda M, Furukawa H, Imamura H, et al: Surgery for gastric cancer increases plasma levels of vascular endothelial growth factor and von Willebrand factor. Gastric Cancer 5: 137-141, 2002.

19. Furuta K, Kakita A, Takahashi T, et al: Experimental study on liver regeneration after simultaneous partial hepatectomy and pancreatectomy. Hepatol Res 17: 223-236, 2000.

20. Tang TX, Hashimoto T, Chao LY, et al: Effects of partial pancreatectomy on liver regeneration in rats. J Surg Res 72: 8-14, 1997.

21. Weese JL, Ottery FD and Emoto SE: Do operations facilitate tumor growth? An experimental study in rats. Surgery 100: 273-277, 1986 .

22. Wildbrett $\mathrm{P}, \mathrm{Oh} \mathrm{A}, \mathrm{Carter} \mathrm{JJ}$, et al: Increased rates of pulmonary metastases following sham laparotomy compared to $\mathrm{CO} 2$ pneumoperitoneum and the inhibition of metastases utilizing perioperative immunomodulation and a tumor vaccine. Surg Endosc 16: 1162-1169, 2002.
23. Allendorf JD, Bessler M, Kayton ML, et al: Increased tumor establishment and growth after laparotomy vs laparoscopy in a murine model. Arch Surg 130: 649-653, 1995.

24. DaCosta ML, Redmond HP, Finnegan N, et al: Laparotomy and laparoscopy differentially accelerate experimental flank tumor growth. Br J Surg 85: 1439-1442, 1998.

25. Kirman I, Cedik V, Poltaratskaia N, et al: Plasma from patients undergoing major open surgery stimulates in vitro tumor growth: lower insulin-like growth factor binding protein 3 levels may, in part, account for this change. Surgery 132: 186-192, 2002.

26. Kirman I, Cedik V, Poltaratskaia N, et al: Open surgery induces a dramatic decrease in circulating intact IGFBP-3 in patients with colorectal cancer not seen with laparoscopic surgery. Surg Endosc 19: 55-59, 2005.

27. Wu FPK, Westphal JR, Hoekman K, et al: The effects of surgery, with and without rh GMCSF, on the angiogenic profile of patients treated for colorectal carcinoma. Cytokine 25: 68-72, 2004.

28. Fausto N: Liver regeneration. J Hepatol 32: 19-31, 2000.

29. Namekata K, Takamori S, Kojima, et al: Significant changes in the serum levels of IL-6, h-HGF and type IV collagen during the perioperative period of a hepatectomy: relevance to SIRS. Surg Today 30: 403-409, 2000

30. Tomiya T, Ogata I and Fujiwara K: Transforming growth factor levels in liver and blood correlate better than hepatocyte growth factor with hepatocyte proliferation during liver regeneration. Am J Pathol 153: 955-961, 1998.

31. Meredith K, Haemmerich D, Qi C and Mahvi D: Hepatic resection but not radiofrequency ablation results in tumor growth and increased growth factor expression. Ann Surg 245: 771-776, 2007. 